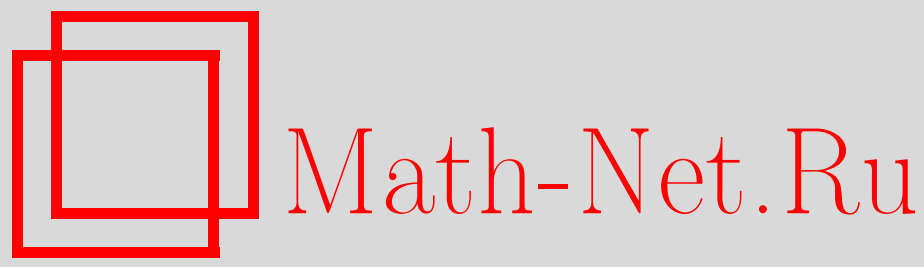

Х. Гомес-Монт, П. Мардесич, Индекс векторного поля, касательного к нечетномерной гиперповерхности, и сигнатура относительного гессиана, Функи. анализ и его прил., 1999, том 33, выпуск 1, 1-13

DOI: https://doi.org/10.4213/faa334

Использование Общероссийского математического портала MathNet.Ru подразумевает, что вы прочитали и согласны с пользовательским соглашением

http://www.mathnet.ru/rus/agreement

Параметры загрузки:

IP : 35.173 .219 .12

26 апреля 2023 г., 10:36:19

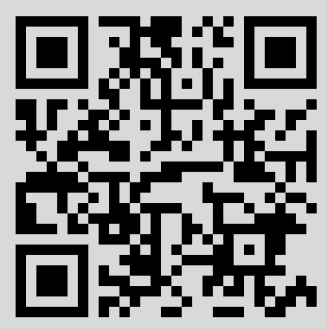


Функииональный анализ и его приложения

1999, т. 33, вып. 1, с. 1-13

УдК $512.774,514.742 .4$

\title{
Индекс векторного поля, касательного к нечетномерной гиперповерхности, и сигнатура относительного гессиана
}

\author{
(c) $1999 . \quad$ Х. Гомес-Монт, П. МАРДЕСИч
}

Пусть $f$ - вещественно-аналитическая функция, определенная на единичном шаре $B$ в $\mathbb{R}^{n+1}$ с естественной ориентацией, и предположим, что $f$ имеет алгебраически изолированную особенность в 0 . Это означает, что комплексификация функции $f$ имеет изолированную особенность в начале координат. Предположим, что $f(0)=0$, и рассмотрим вещественную гиперповерхность $V:=f^{-1}(0) \subset B \subset \mathbb{R}^{n+1}$. Обозначим через $\mathfrak{X}(V)$ множество ростков вещественно-аналитических векторных полей в $0 \in B$, касательных к $V$ и имеющих алгебраически изолированную особенность в начале координат.

В [8] и [3] было введено геометрическое понятие индекса (Пуанкаре-Хопфа) $\operatorname{Ind}_{V, \pm, 0}(X)$ векторного поля $X$, касательного к гиперповерхности $V$, для векторных полей, имеющих изолированную особенность в $0 \in V$,

$$
\operatorname{Ind}_{V, \pm, 0}: \mathfrak{X}(V) \rightarrow \mathbb{Z} .
$$

По определению индекс - это сумма индексов Пуанкаре-Хопфа особых точек векторного поля $X_{ \pm}$на сглаживании $V_{ \pm}:=f^{-1}( \pm \varepsilon)$, где $\varepsilon>0$ мало, до вырождения $\left(X_{ \pm}, V_{ \pm}\right)$к $(X, V)$.

Сопоставим функции $f$ локальную алгебру

$$
\mathbf{A}=\frac{\mathscr{A}_{B, 0}}{\left(f_{0}, \ldots, f_{n}\right)}, \quad f_{i}=\frac{\partial f}{\partial x_{i}},
$$

где $\mathscr{A}_{B, 0}$ - кольцо ростков вещественно-аналитических функций в $0 \in \mathbb{R}^{n+1}$. Вещественно-аналитическому полю $X \in \mathfrak{X}(V)$ мы сопоставим локальную алгебру

$$
\mathbf{B}=\frac{\mathscr{A}_{B, 0}}{\left(X^{0}, \ldots, X^{n}\right)}, \quad X=\sum_{i=0}^{n} X^{i} \frac{\partial}{\partial x_{i}} .
$$

Из предположений, что начало координат является алгебраически изолированной особенностью функции $f$ и векторного поля $X$, вытекает, что обе эти алгебры являются конечномерными $\mathbb{R}$-векторными пространствами. В каждой из алгебр $\mathbf{A}$ и $\mathbf{B}$ имеется выделенный элемент - класс гессиана функции $f$ и класс якобиева определителя векторного поля $X$

$$
\operatorname{Hess}(f):=\operatorname{det}\left[\frac{\partial^{2} f}{\partial x_{i} \partial x_{j}}\right] \in \mathbf{A}, \quad J X:=\operatorname{det}[D X]=\operatorname{det}\left[\frac{\partial X^{j}}{\partial x_{k}}\right] \in \mathbf{B} .
$$

Они являются образующими цоколей соответствующих алгебр (т.е. минимальных ненулевых идеалов, размерности которых над $\mathbb{R}$ равны единице; 
cр. $[5,10])$. Предположение, что $X$ касается $V$, дает равенство

$$
d f(X)=\sum_{j=0}^{n} f_{j} X^{j}=f h_{X}, \quad h_{X} \in \mathscr{A}_{B, 0} .
$$

Рассмотрим класс элемента $h:=h_{X}$ в алгебрах А и В. В [7] мы определили относительныи якобиан $J_{f} X:=J X / h \in \mathbf{B} / \operatorname{Ann}_{\mathbf{B}}(h)$, если класс элемента $h$ не равен нулю в $\mathbf{B}$. Точно так же мы определяем относительный гессиан

$$
\operatorname{Hess}_{\text {rel }}(h):=\frac{\operatorname{Hess}(f)}{h} \in \frac{\mathbf{A}}{\operatorname{Ann}_{\mathbf{A}}(h)},
$$

если класс элемента $h$ не равен нулю в $\mathbf{A}$. Относительный гессиан и якобиев определитель порождают цоколи алгебр $\mathbf{A} / \operatorname{Ann}_{\mathbf{A}}(h)$ и $\mathbf{B} / \operatorname{Ann}_{\mathbf{B}}(h)$ соответственно [7]. Согласно общей теории (см. $[2,5,10])$ строятся невырожденные спаривания

$$
\begin{array}{ll}
\frac{\mathbf{A}}{\operatorname{Ann}_{\mathbf{A}}(h)} \times \frac{\mathbf{A}}{\operatorname{Ann}_{\mathbf{A}}(h)} \stackrel{\dot{\rightarrow}}{\rightarrow} \frac{\mathbf{A}}{\operatorname{Ann}_{\mathbf{A}}(h)} \stackrel{\ell}{\rightarrow} \mathbb{R}, & \ell\left(\operatorname{Hess}_{\mathrm{rel}}(h)\right)>0, \\
\frac{\mathbf{B}}{\operatorname{Ann}_{\mathbf{B}}(h)} \times \frac{\mathbf{B}}{\operatorname{Ann}_{\mathbf{B}}(h)} \stackrel{\dot{\mathbf{B}}}{\rightarrow} \frac{\mathbf{B}}{\operatorname{Ann}_{\mathbf{B}}(h)} \stackrel{\ell^{\prime}}{\rightarrow} \mathbb{R}, & \ell^{\prime}\left(J_{f} X\right)>0,
\end{array}
$$

сигнатуры которых не зависят от выбора линейных функций $\ell$ и $\ell^{\prime}$. Определим функиии относительных сигнатур

$$
\operatorname{Sgn}_{\mathbf{A}}: \mathbf{A} \rightarrow \mathbb{Z}, \quad \operatorname{Sgn}_{V, 0}: \mathfrak{X}(V) \rightarrow \mathbb{Z},
$$

где $\operatorname{Sgn}_{\mathbf{A}}(h)$ - сигнатура спаривания (1), если класс элемента $h$ не равен нулю в $\mathbf{A}$, и $\operatorname{Sgn}_{\mathbf{A}}(0)=0$. Аналогично [7], $\operatorname{Sgn}_{V, 0}(X)$ - сигнатура спаривания (2), если класс элемента $h$ не равен нулю в $\mathbf{B}$, и $\operatorname{Sgn}_{V, 0}(X)=0$ в противном случае.

Теорема 1. Пусть $n \geqslant 1$ - нечетное натуральное число и $V=f^{-1}(0) \subset$ $\mathbb{R}^{n+1}$ - вещественнал гиперповерхность с алгебраччески изолированной особенностью в начале координат. Тогда функиия индекса (Пуанкаре-Xопфа) $\operatorname{Ind}_{V, \pm, 0}$ в $0-э m o$

$$
\operatorname{Ind}_{V, \pm, 0}(X)=\operatorname{Sgn}_{V, 0}(X)-\operatorname{Sgn}_{\mathbf{A}}\left(\frac{d f}{f}(X)\right) \quad \forall X \in \mathfrak{X}(V) .
$$

Функции сигнатур $\operatorname{Sgn}_{V, 0}$ u $\operatorname{Sgn}_{\mathbf{A}}$ удовлетворяют закону сохранения:

$$
\begin{aligned}
& {\left[\operatorname{Sgn}_{V, 0}(X)-\operatorname{Sgn}_{\mathbf{A}}\left(\frac{d f}{f}(X)\right)\right]=} {\left[\operatorname{Sgn}_{V, 0}\left(X_{t}\right)-\operatorname{Sgn}_{\mathbf{A}}\left(\frac{d f}{f}\left(X_{t}\right)\right)\right] } \\
&+\sum_{\substack{X_{t}(p)=0, f(p)=0, p \in \mathbb{R}^{n} \backslash\{0\}}} \operatorname{Ind}_{V, p}\left(X_{t} \mid V\right),
\end{aligned}
$$

где $X$ и $X_{t}$ - вещественно-аналитические векторные поля, касательные $\kappa V, X_{t}$ близко $\kappa X, p$ близко $\kappa 0 u \operatorname{Ind}_{V, p}\left(\left.X_{t}\right|_{V}\right)$ обозначает индекс Пуанкаре-Хопфа векторного поля $\left.X_{t}\right|_{V}$ в неособой точке $p \in V \backslash\{0\}$. 
Доказательство теоремы 1 будет проведено в три шага:

1) Формула (5) доказывается при условии, что размерность идеала $\left(h_{X_{t}}\right) \subset$ A не зависит от $t$ (предложение 3.1 с использованием [7]).

2) Граница между стратами, отвечающими постоянной размерности идеала, пересекается в точке, соответствующей гамильтонову векторному полю $H$, вдоль семейства $H+t Y$ (предложение 4.1). Это доказывает (5) в общем случае.

3) Формула (5) влечет за собой (4) с точностью до аддитивной постоянной. Снова оценивая эту постоянную для гамильтонова векторного поля, мы доказываем, что она равна 0 .

Геометрическая интерпретация, отвечающая формуле (4), описывается следуюшим образом. Имеются два типа особых точек векторного поля $X_{t}$ :

а) особые точки, захваченные в $\mathbf{B}_{t} / \operatorname{Ann}_{\mathbf{B}_{t}}\left(h_{t}\right)$ и измеряемые относительным якобианом;

b) особые точки из $\operatorname{Spec} \mathbf{A}$, которые не принадлежат $\{X=0\}$ и которые измеряются относительным гессианом в $\mathbf{A} / \operatorname{Ann}_{\mathbf{A}}\left(h_{t}\right)$.

Подсчитываются точки первого типа минус точки второго типа. Этот способ подсчета специально приспособлен для случая нечетного $n$. Для четного $n$ см. [7]. Гамильтоново векторное поле является правильным местом для «пересечения границы», поскольку множество его особых точек не содержит точек первого класса, но содержит все точки второго класса, т. е. никакие точки из Spec $\mathbf{A}$ не теряются. Для векторного поля $H+t Y$ мы в предложении 4.1 показываем, что точки второго класса из $\mathbf{A} / \operatorname{Ann}_{\mathbf{A}}((d f / f)(Y))$ для $t=0$ перемещаются из Spec $\mathbf{A}$ в точки второго класса со знаками из $\mathbf{B}_{t} / \operatorname{Ann}_{\mathbf{B}_{t}}(h)$ при $t \neq 0$.

Упомянем также недавнюю родственную работу об индексе градиентного векторного поля на полном пересечении [4].

\section{§1. Сигнатуры, определенные гессианом}

С помощью гессиана $\operatorname{Hess}(f)$ pocтка $f$, взяв $h=1$, мы определяем невырожденное билинейное спаривание на конечномерной алгебре $\mathbf{A}$. Пусть $\sigma-$ сигнатура этой билинейной формы. Следующий результат В. И. Арнольда $[1$, c. 3] описывает геометрический смысл $\sigma$ :

ПРЕДЛОЖЕНИЕ 1.1. Пусть $B \subset \mathbb{R}^{n+1}$ - достаточно маленький шар, так что гиперповерхность $V$ стягиваема, и обозначим через $\chi^{+}$и $\chi^{-}$эйлеровь характеристики многообразий (с границами) $V_{\varepsilon}:=f^{-1}(\varepsilon)$ u $V_{-\varepsilon}$ соответственно, $\varepsilon>0$ мало. Пусть $L:=V \cap \partial B$ - зачепление гиперповерхности $\checkmark$ на сфере $ә В$. Тогда

(i) сигнатура $\sigma$ является индексом Пуанкаре-Хопфа градиентного потока $\sum_{j} f_{j} \cdot \partial / \partial x_{j}$ на $B$ в начале координат 0 ;

(ii) для нечетного $n$ имеем

$$
\chi^{+}=\chi^{-}=1-\sigma, \quad \chi(L)=2(1-\sigma) ;
$$

(iii) для четного $n$ имеем

$$
\chi^{+}=1+\sigma, \quad \chi^{-}=1-\sigma, \quad \chi^{+}-\chi^{-}=2 \sigma .
$$


Гессиан определяет образующую цоколя кольца А, выбирая что-то вроде ориентации на $\mathbf{A}$, а затем определяет невырожденную двойственность (1). Гессиан будет также определять такую ориентацию и невырожденную двойственность на семействах алгебр $\left\{\mathbf{A} / \operatorname{Ann}_{\mathbf{A}}(h)\right\}_{h \in A}$, если определить относительный гессиан $\operatorname{Hess}(f) / h \in \mathbf{A} / \operatorname{Ann}_{\mathbf{A}}(h)$ и заметить, что он является образующей цоколя в этой факторалгебре [7]. Следовательно, с его помощью мы можем определить невырожденное билинейное спаривание в $\mathbf{A} / \operatorname{Ann}_{\mathbf{A}}(h)$, как в (1). Если варьировать $h_{t} \in \mathbf{A}$ таким образом, что ранг идеала $\left(h_{t}\right) \subset \mathbf{A}$ остается постоянным, то (поскольку спаривание невырожденно) сигнатуры билинейных форм остаются постоянными, покуда пространство параметров связно. Заметим, что сигнатура $\sigma$ в предложении 1.1 (определенная, как в (3)) равна $\operatorname{Sgn}_{\mathbf{A}}(1)$.

Удобно ввести геометрический язык. Spec A геометрически состоит из одной точки 0 с алгеброй функций А. Мы представляем ее как «жирную точку», состоящую из $\operatorname{dim} \mathbf{A}$ (обычных) точек, слившихся вместе в начале координат образом, определенным структурой алгебры $\mathbf{A}$. Тогда семейство алгебр $\left\{\mathbf{A} / \operatorname{Ann}_{\mathbf{A}}(h)\right\}$ описывает семейство подточек

$$
\left\{\operatorname{Spec} \frac{\mathbf{A}}{\operatorname{Ann}_{\mathbf{A}}(h)} \subset \operatorname{Spec} \mathbf{A}\right\}_{h \in \mathbf{A}} .
$$

Эти подточки определяются уравнениями, содержащимися в $\operatorname{Ann}_{A}(h)$. Kaждая подточка семейства обладает невырожденным спариванием, определенным выбором относительного гессиана $\operatorname{Hess}(f) / h$ в качестве образующей цоколя. Поскольку нас в основном интересует не функция $f$, а пространство $V=f^{-1}(0)$, мы занимаемся кольцом $\mathbf{A} /(f)$, а указанное выше семейство, ограниченное на $h \in \operatorname{Ann}_{\mathbf{A}}(f)$, даст семейство подточек

$$
\left\{\operatorname{Spec} \frac{\mathbf{A}}{\operatorname{Ann}_{\mathbf{A}}(h)} \subset \operatorname{Spec} \frac{\mathbf{A}}{(f)}\right\}_{h \in \operatorname{Ann}_{\mathbf{A}}(f)} .
$$

Каждая из этих подточек обладает невырожденным спариванием, и ее сигнатура определяет функцию

$$
\operatorname{Sgn}_{\mathbf{A}}: \operatorname{Ann}_{\mathbf{A}}(f) \rightarrow \mathbb{Z}
$$

которая ставит в соответствие элементу $h \in \mathrm{Ann}_{\mathbf{A}}(f)$ сигнатуру билинейной формы, определенной на $\mathbf{A} / \operatorname{Ann}_{\mathbf{A}}(h)$ относительным гессианом. Чтобы понять природу функции (1.1), рассмотрим отображение

$$
\operatorname{Ann}_{\mathbf{A}}(f) \times \mathbf{A} \rightarrow \operatorname{Ann}_{\mathbf{A}}(f) \times \mathbf{A}, \quad(h, g) \rightarrow(h, h \cdot g) .
$$

Его можно рассматривать как семейство эндоморфизмов конечномерного векторного пространства $\mathbf{A}$, параметризованное элементами $h \in \operatorname{Ann}_{\mathbf{A}}(f)$, и, следовательно, отображение (1.2) можно рассматривать как семейство матриц, параметризованное элементами $h \in \operatorname{Ann}_{\mathbf{A}}(f)$. Ранг матрицы, соответствующей ростку $h$, равен размерности идеала $(h) \subset \mathbf{A}$. Мы получаем стратификацию пространства параметров $\operatorname{Ann}_{\mathbf{A}}(f)$ в виде объединения конечного числа полуаналитических подмножеств, являющихся связными компонентами подпространств

$$
\{h \in \mathbf{A} / \operatorname{dim}(h)=k\} .
$$


ЛЕмма 1.2. Пусть $V_{1}, \ldots, V_{r}$ - страты указанного выше разложения пространства $\operatorname{Ann}_{\mathbf{A}}(f)$ на связнье компоненты множеств постоянного ранга идеала $(h) \subset \mathbf{A}$. Тогда функиия $\operatorname{Sgn}_{\mathbf{A}}$ постоянна на каждом из множеств $V_{j}$.

ДоказАтельство. Ограничивая $h \in \operatorname{Ann}_{\mathbf{A}}(f)$ на один из стратов, мы получаем семейство алгебр $\mathbf{A} / \operatorname{Ann}_{\mathbf{A}}(h)$ постоянной размерности, и, выбирая непрерывное семейство линейных функций $\ell_{t}$, таких, что $\ell_{t}\left(\operatorname{Hess}(f) / h_{t}\right)>0$, приходим к непрерывному семейству невырожденных билинейных форм $[5,10]$, сигнатуры которых остаются постоянными вдоль каждого страта.

Информация, содержащаяся в функции $\operatorname{Sgn}_{\mathbf{A}}$, определяется целым числом, ассоциированным с каждым стратом $V_{j}$; при этом элементу $h=0$ ставится в соответствие целое число 0. Заметим, что, поскольку $h \in \operatorname{Ann}_{\mathbf{A}}(f)$, отображение $\mathbf{A} \rightarrow \mathbf{A}$, получаемое умножением элементов из $\mathbf{A}$ на $h$, естественным образом индуцирует отображение $h: \mathbf{A} /(f) \rightarrow \operatorname{Ann}_{\mathbf{A}}(f)$. Тогда гомоморфизм (1.2) индуцирует отображение

$$
\operatorname{Ann}_{\mathbf{A}}(f) \times \frac{\mathbf{A}}{(f)} \rightarrow \operatorname{Ann}_{\mathbf{A}}(f) \times \operatorname{Ann}_{\mathbf{A}}(f) .
$$

Если рассматривать это отображение как семейство матриц с параметрами из пространства $\operatorname{Ann}_{\mathbf{A}}(f)$, то стратификация пространства параметров $\operatorname{Ann}_{\mathbf{A}}(f)$ на связные компоненты множеств постоянного ранга этого семейства матриц совпадает с такой же стратификацией, определяемой семейством (1.2).

Пример 1.3. Пусть $f=y^{3}-x^{2}$ - функция на $\mathbb{R}^{2}$. Тогда $\mathbf{A}=\mathbb{R} \oplus \mathbb{R} y$, $\operatorname{Hess}(f)=-12 y$. Билинейная форма, индуцированная гессианом на $\mathbf{A}$, имеет сигнатуру $\sigma=0$. Имеем $f=0 \in \mathbf{A}, \operatorname{Ann}_{\mathbf{A}}(f)=\mathbf{A}$, а идеалы $(h) \subset \mathbf{A}$ бывают трех типов: $(0),(y),(1)$, имеющих ранги 0,1 и 2 соответственно. Стратификация пространства $\operatorname{Ann}_{\mathbf{A}}(f)=\mathbf{A}$ определяется следующим образом:

1) Ранг 2. Соответствующие страты состоят из единиц кольца $\mathbf{A}$ - элементов вида $a+b y$ с $a \neq 0$. Имеются два страта, определяемые знаком $a$. Относительный гессиан равен $(-12 / a) y \in \mathbf{A} / \operatorname{Ann}_{\mathbf{A}}(h)=\mathbf{A}$, и сигнатура равна нулю для обоих стратов.

2) Ранг 1. Имеются два страта, состояшие из элементов вида $\{b y\}$, c $b>0$ для одного из них и $b<0$ для другого. Имеем

$$
\frac{\mathbf{A}}{\operatorname{Ann}_{\mathbf{A}}(b y)} \simeq \frac{\mathbf{A}}{(y)} \simeq \mathbb{R},
$$

а относительный гессиан равен $-12 / b$. Следовательно, сигнатура равна -1 на положительной полуоси $b>0$ и +1 на отрицательной полуоси $b<0$.

3) Ранг 0. Страт состоит из единственной точки 0 , и сигнатура равна 0 .

Таким образом, в этом примере функция сигнатуры $\operatorname{Sgn}_{\mathbf{A}}$ определяется формулой

$$
\operatorname{Sgn}_{A}(a+b y)=\left\{\begin{array}{cl}
-1, & a=0, b>0 \\
+1, & a=0, b<0 \\
0 & \text { в противном случае. }
\end{array}\right.
$$




\section{§2. Отображение вычета $\delta_{f}$}

Пусть, как и ранее, $f$ - вещественно-аналитическая функция с алгебраически изолированной особенностью в начале координат. Обозначим через $\Theta_{B, 0}$ пространство ростков вещественно-аналитических векторных полей $X=\sum_{k=0}^{n} X^{k} \partial / \partial x_{k}$ в $0 \in B \subset \mathbb{R}^{n+1}$, а через $\Theta_{B, V, 0}$ - пространство ростков вещественно-аналитических векторных полей в 0 , касающихся гиперповерхности $V=f^{-1}(0)$. Условие того, что векторное поле $X$ касается $V$, записывается в виде

$$
d f(X)=\sum_{j=0}^{n} f_{j} X^{j}=f h_{X}, \quad h_{X} \in \mathscr{A}_{B, 0},
$$

и, следовательно, имеется корректно определенное $\mathscr{A}_{B, 0}$-линейное отображение

$$
\frac{d f}{f}: \Theta_{B, V, 0} \rightarrow \mathscr{A}_{B, 0}, \quad X \rightarrow h_{X}=\frac{d f}{f}(X) .
$$

Определим отображение вычета как $\mathscr{A}_{B, 0}$-линейное отображение, получаемое из указанного выше факторизацией образа по идеалу, порожденному частными производными $\left(f_{0}, \ldots, f_{n}\right)$ :

$$
\delta_{f}:=\frac{d f}{f}: \Theta_{B, V, 0} \rightarrow \mathbf{A}=\frac{\mathscr{A}_{B, 0}}{\left(f_{0}, \ldots, f_{n}\right)} .
$$

ПРЕДЛОЖЕНИЕ 2.1. 1) Образ отображения $\delta_{f}$ совпадает с $\operatorname{Ann}_{\mathbf{A}}(f)$.

2) $\delta_{f}(X)$ зависит только от ограничения векторного поля $X$ на $V u$, следовательно, индуцирует отображение

$$
\delta_{V}: \Theta_{V, 0} \rightarrow \operatorname{Ann}_{\mathbf{A}}(f) .
$$

3) $\delta_{V}(X)=0$ тогда и только тогда, когда существует аналитическое продолжение векторного поля $\left.X\right|_{V}$ на окрестность начала координат $0 \in$ $\mathbb{R}^{n+1}$, касательное $\kappa$ слоям $\left\{f^{-1}(t)\right\}$ ростка $f$.

ДокАЗАТЕЛЬСТво. В соответствии с (2.1) $f \delta_{f}(X)$ представлена ростком $\sum_{j=0}^{n} f_{j} X^{j}$, который равен 0 в $\mathbf{A}$. Следовательно, $\delta_{f}\left(\Theta_{B, V, 0}\right) \subset \operatorname{Ann}_{\mathbf{A}}(f)$. Наоборот, если $h \in \operatorname{Ann}_{\mathbf{A}}(f)$, то $h f=0 \in \mathbf{A}$. Это означает, что $h f=$ $\sum_{j=0}^{n} f_{j} X^{j}$ для некоторых $X^{0}, \ldots, X^{n}$ и $h=\delta_{f}(X)$ для $X=\sum_{k=0}^{n} X^{k} \partial / \partial x_{k}$. Это доказывает ч. 1).

Пусть $X$ и $Y$ - два векторных поля из $\Theta_{B, V, 0}$, ограничения которых на $V$ совпадают. Тогда $Y=X+f Z$ для некоторого $Z \in \Theta_{B, 0}$. Однако $\delta_{f}(f Z)=0$, поскольку $(d f / f)(f Z)=d f(Z) \in\left(f_{0}, \ldots, f_{n}\right)$. Это доказывает 2).

Чтобы доказать 3$)$, заметим, что векторное поле $W$ касается слоев ростка $f$, если и только если $d f(W)$ тождественно равно нулю. Все продолжения векторного поля $X \in \Theta_{V, 0}$ имеют вид $X+f Y$, где, если допустить некоторую некорректность обозначений, $X \in \Theta_{B, V, 0}$ - одно из таких продолжений. Имеем $d f(X+f Y)=f h_{X}+f \sum_{j=0}^{n} f_{j} Y^{j}$. Следовательно, $d f(X+f Y) \equiv 0$ тогда и только тогда, когда

$$
h_{X}=\sum_{j=0}^{n} f_{j} Y^{j}
$$


Таким образом, векторное поле $X$ может быть продолжено на все пространство $\mathbb{C}^{n+1}$ так, что оно будет касаться слоев функции $f$ тогда и только тогда, когда $\delta_{V}(X)=0$. В этом случае такое продолжение имеет вид $X+f Y$, где компоненты векторного поля $Y$ удовлетворяют уравнению $(2.2)$.

\section{§3. Идеал $\left(\delta\left(X_{t}\right)\right) \subset$ А имеет постоянную размерность}

Пусть $X_{t}$ - вещественно-аналитическое семейство вещественно-аналитических векторных полей, имеющих голоморфные продолжения на замкнутый единичный шар $\bar{B}_{\mathbb{C}} \subset \mathbb{C}^{n+1}$, параметризованное связным приведенным аналитическим пространством $T$, такое, что каждое из векторных полей $X_{t}$ имеет изолированные особенности на $\bar{B}_{\mathbb{C}}$ и не обращается в нуль на границе шара $B_{\mathbb{C}}$. Пусть $0 \in T$, и предположим, что векторное поле $X_{0}$ обрашается в нуль только в начале координат. Имеется векторное расслоение, слоями которого являются конечномерные (скажем, $\mu$-мерные) полулокальные алгебры

$$
{ }^{\mathbb{C}} \mathbf{B}_{t}:=H^{0}\left(B_{\mathbb{C}}, \frac{\mathscr{O}_{B_{\mathbb{C}}}}{\left(X_{t}^{0}, \ldots, X_{t}^{n}\right)}\right)=\bigoplus_{\substack{\left\{X_{t}\left(p_{t}\right)=0\right\} \\ p_{t} \in B_{\mathbb{C}}}} \frac{\mathscr{O}_{B_{\mathbb{C}}, p_{t}}}{\left(X_{t}^{0}, \ldots, X_{t}^{n}\right)}:=\bigoplus_{\left\{X_{t}\left(p_{t}\right)=0\right\}} \mathbb{C}_{\mathbf{B}_{t, p_{t}}},
$$

обладающие указанным выше разложением в прямую сумму $\mathbb{C}$-локальных алгебр. Инвариантные подпространства отображения комплексного сопряжения

$$
{ }^{-}:{ }^{\mathbb{C}} \mathbf{B}_{t} \rightarrow{ }^{\mathbb{C}} \mathbf{B}_{t}
$$

определяют на $T$ вещественно-аналитическое векторное расслоение $\mathbb{R}-$-лгебр вещественной размерности $\mu$.

Разложение (3.1) алгебры ${ }^{\mathbb{C}} \mathbf{B}_{t}$ индуцирует разложение в прямую сумму

$$
\left[\mathbf{B}_{t}\right]_{\mathbb{R}}^{+}=\left[\bigoplus_{j} D_{j}\right] \oplus\left[\bigoplus_{k} E_{k}\right]
$$

где каждая компонента $D_{j}$ соответствует вещественной точке из $B:=B_{\mathbb{C}} \cap$ $\mathbb{R}^{n+1}$, а каждая компонента $E_{k}$ соответствует паре комплексно-сопряженных точек из $B_{\mathbb{C}} \backslash B$. Для $p_{j} \neq 0$ и для любого $k$ имеем

$$
D_{j}=\frac{\mathscr{A}_{\mathbb{R}^{n+1}, p_{j}}}{\left(X_{t}^{0}, \ldots, X_{t}^{n}\right)}, \quad E_{k}=\left[\frac{\mathscr{O}_{\mathbb{C}^{n+1}, p_{k}}}{\left(X_{t}^{0}, \ldots, X_{t}^{n}\right)} \oplus \frac{\mathscr{O}_{\mathbb{C}^{n+1}, \bar{p}_{k}}}{\left(X_{t}^{0}, \ldots, X_{t}^{n}\right)}\right]_{\mathbb{R}}^{+} .
$$

Для любого вещественно-аналитического семейства $\mathbb{R}$-линейных отображений $\ell_{t}:\left[\mathbf{B}_{t}\right]_{\mathbb{R}}^{+} \rightarrow \mathbb{R}$, таких, что $\ell_{0}\left(J X_{0}\right)>0$, билинейные формы из вешественно-аналитического семейства $\left\langle e_{t}, k_{t}\right\rangle_{t}:=\ell_{t}\left(e_{t} k_{t}\right)$ невырожденны для $t$, близких к $0 \in T$.

Ограничение описанной билинейной формы на компоненту $D_{j}$, соответствующую точке $p \in B \backslash\{0\}$, совпадает с билинейной формой АйзенбадаЛевина-Химшиашвили $[5,10]$ векторного поля $X_{t}$ в точке $p$, а ее сигнатура равна индексу Пуанкаре-Хопфа векторного поля $X_{t}$ в точке $p$. Ограничение этой билинейной формы на компоненту $E_{k}$, соответствующую паре комплексно-сопряженных точек из $B_{\mathbb{C}} \backslash B$, имеет сигнатуру, равную 0. Из 
непрерывности невырожденных билинейных форм вытекает фундаментальный закон сохранения для индекса Пуанкаре-Хопфа:

$$
\operatorname{Ind}_{\mathbb{R}^{n+1}, q}\left(X_{t_{0}}\right)=\sum_{\substack{X_{t}(p)=0, p \in B,|p-q|<\varepsilon}} \operatorname{Ind}_{\mathbb{R}^{n+1}, p}\left(X_{t}\right) .
$$

Сигнатура $\operatorname{Sgn}_{V, 0}\left(X_{t}\right)$ также удовлетворяет закону сохранения для семейства $X_{t}$ при условии, что идеал в алгебре $\mathbf{A}$, порожденный $f$-вычетом $\delta_{f}\left(X_{t}\right)$, имеет постоянную размерность:

ПрЕДЛОЖЕНИЕ 3.1. Пусть $\left\{X_{t}\right\}_{t \in T}$ - параметризованное связным пространством $T$ вещественно-аналитическое семейство вещественно-аналитических векторных полей на шаре $B$, касающихсл $V$ и имеюших алгебраически изолированную особую точку в начале координат $0 \in B$, и пусть $\left\{V_{i}\right\}$ - связнье страты пространства $\operatorname{Ann}_{\mathbf{A}}(f)$ из леммы 1.2. Предположим, что для всех $t \in T$ f-вычет $\delta_{f}\left(X_{t}\right)$ лежит в биксированном страте $V_{i_{0}}$. Тогда любой элемент $X_{t_{0}}$ рассматриваемого семейства $\left(t_{0} \in T\right)$ удовлетворяет закону сохранения

$$
\operatorname{Sgn}_{V, 0}\left(X_{t_{0}}\right)=\operatorname{Sgn}_{V, 0}\left(X_{t}\right)+\sum_{\substack{X_{t}(p)=0, f(p)=0, p \in \mathbb{R}^{n} \backslash\{0\}}} \operatorname{Ind}_{V, p}\left(\left.X_{t}\right|_{V}\right)
$$

для $t \in T$, близкого $\kappa t_{0} \in T, u p$, близких $\kappa$ началу координат 0 .

ДокАЗАтЕльство. Из предположения, что $\delta_{f}\left(X_{t}\right) \in V_{i_{0}}$, следует, что размерность идеала $\left(h_{t}\right):=\left((d f / f)\left(X_{t}\right)\right) \subset \mathbf{A}$ постоянна для $t \in T$. Из теоремы 2.4 работы $[6]$ мы получаем изоморфизм $\mathbb{R}$-алгебр

$$
\frac{\operatorname{Ann}_{\mathbb{A}} \mathbf{A}(f)}{\left(h_{t}\right)} \simeq \frac{\operatorname{Ann}_{\mathbf{B}_{t}}(f)}{\left(h_{t}\right)},
$$

где ${ }^{\mathbb{C}} \mathbf{A}:=\mathbf{A} \otimes_{\mathbb{R}} \mathbb{C}$. В силу теоремы 3 из [6] для четного $n$ семейство ${ }^{\mathbb{C}} \mathbf{B}_{t} /\left(h_{t}\right)$ является $t$-плоским (т. е. соответствующая размерность не зависит от $t$ ), а для нечетного $n t$-плоским является семейство ${ }^{\mathbb{C}} \mathbf{B}_{t} /(f)$. Для четного $n$, используя (3.3), мы получаем, что семейство ${ }^{\mathbb{C}} \mathbf{B}_{t} / \operatorname{Ann}_{\mathbf{B}_{t}}(f)$ является $t$-плоским. Поскольку $(f)$ и $\operatorname{Ann}_{\mathbf{B}_{t}}(f) \subset{ }^{\mathbb{C}} \mathbf{B}_{t}$ имеют дополнительные размерности, а семейство ${ }^{\mathbb{C}} \mathbf{B}_{t}$ является $t$-плоским, семейство ${ }^{\mathbb{C}} \mathbf{B}_{t} /(f)$ также является $t$-плоским. Следовательно, семейство ${ }^{\mathbb{C}} \mathbf{B}_{t} /(f)$ является $t$-плоским для обеих четностей. Это условие требуется для применимости теоремы 6 из [7], и поэтому из части (v) той теоремы мы получаем (3.2).

\section{$\S 4$. Индекс векторного поля на нечетномерной гиперповерхности}

Пусть $n \geqslant 1$ - положительное нечетное число, и пусть $X_{t}$ и ${ }^{\mathbb{C}} \mathbf{B}_{t}$ такие же, как в (3.1). В силу теоремы 3 из [6] для нечетного $n$ семейство идеалов $(f) \subset$ ${ }^{\mathbb{C}} \mathbf{B}_{t}$ является векторным подрасслоением, как и двойственное к нему семейство $\operatorname{Ann}_{\mathbf{B}_{t}}(f) \subset{ }^{\mathbb{C}} \mathbf{B}_{t}$. Мы получаем отображение векторных расслоений

$$
\left\{\frac{{ }^{\mathbb{C}} \mathbf{B}_{t}}{(f)}\right\} \stackrel{h_{t}}{\longrightarrow}\left\{\operatorname{Ann}_{\mathbb{C}} \mathbf{B}_{t}(f)\right\} .
$$


Для фиксированного значения $t$ описанное отображение может быть дополнено до точной последовательности

$$
0 \rightarrow \frac{\operatorname{Ann}_{\mathbb{C}_{\mathbf{B}_{t}}}\left(h_{t}\right)}{(f)} \rightarrow \frac{{ }^{\mathbb{C}} \mathbf{B}_{t}}{(f)} \stackrel{h_{t}}{\rightarrow} \operatorname{Ann}_{\mathbb{C}_{\mathbf{B}_{t}}}(f) \rightarrow \frac{\operatorname{Ann}_{\mathbf{B}_{t}}(f)}{\left(h_{t}\right)} \rightarrow 0
$$

Из (3.3) и невырожденности двойственности в $\mathbf{B}_{t}$ следует, что комплексная размерность двух крайних векторных пространств в последовательности (4.1) равна $\operatorname{dim}_{\mathbb{R}}\left(\operatorname{Ann}_{\mathbf{A}}(f) /\left(h_{t}\right)\right)$. Первая короткая точная последовательность в (4.1) совпадает с

$$
0 \rightarrow \frac{\operatorname{Ann}_{\mathbf{B}}\left(h_{t}\right)}{(f)} \rightarrow \frac{{ }^{\mathbb{C}} \mathbf{B}_{t}}{(f)} \rightarrow \frac{{ }^{\mathbb{C}} \mathbf{B}_{t}}{\operatorname{Ann}_{\mathbb{C}_{\mathbf{B}_{t}}}\left(h_{t}\right)} \rightarrow 0
$$

Относительный якобиан $J_{f} X_{t}:=J X_{t} / h_{t}$ является элементом последней алгебры в (4.2) и индуцирует невырожденную двойственность на инвариантной относительно комплексного сопряжения части $\left[{ }^{\mathbb{C}} \mathbf{B}_{t} / \operatorname{Ann}_{\mathbb{C}} \mathbf{B}_{t}\left(h_{t}\right)\right]_{\mathbb{R}}^{+} \cdot$ Из предложения 3.1 вытекает непрерывная зависимость от $t$ билинейных форм на $\left[{ }^{\mathbb{C}} \mathbf{B}_{t} / \mathrm{Ann}_{\mathbf{B}_{t}}\left(h_{t}\right)\right]_{\mathbb{R}}^{+}$, определенных относительным якобиевым определителем, при условии, что $\delta_{f}\left(X_{t}\right)$ остается в фиксированном страте $V_{i_{0}}$ пространства A.

Поскольку средний член в точной последовательности (4.2) образует векторное расслоение, падение размерности коядра в (4.2) происходит одновременно с соответствующим скачком размерности ядра в (4.2). Нам следует проверить, что величина сигнатуры, потерянная из-за уменьшения размерности алгебры $\left[{ }^{\mathbb{C}} \mathbf{B}_{t} / \operatorname{Ann}_{\mathbb{C}} \mathbf{B}_{t}\left(h_{t}\right)\right]_{\mathbb{R}}^{+}$, соответствует такой же величине сигнатуры, приобретенной из-за увеличения размерности $\operatorname{Ann}_{\mathbf{B}_{t}}\left(h_{t}\right) /(f) \simeq$ $\operatorname{Ann}_{\mathbf{A}}\left(h_{t}\right) /(f)$. Гамильтоново векторное поле $H$ предоставляет удобное место для «пересечение границы» страта постоянной размерности в лемме 1.2. Заметим, что эта стратификация содержит $0=\delta_{f}(H)$ в качестве страта и что любой другой страт инвариантен относительно умножения на положительные вешественные числа. Элемент $t h=\delta_{f}(H+t Y) \in \operatorname{Ann}_{\mathbf{A}}(f) \subset \mathbf{A}$ с $t \in[0,1]$ содержится в страте $\{0\}$ при $t=0$ и в страте, содержащем $\delta_{f}(Y)$, при $t \neq 0$.

ПРЕДЛОЖЕНИЕ 4.1. Пусть $f$ - вещественно-аналитическая функиия $c$ алгебраически изолированной особой точкой в начале координат 0. Обозначим через

$$
H=\sum_{j=1}^{k}\left[f_{2 j-1} \frac{\partial}{\partial x_{2 j-2}}-f_{2 j-2} \frac{\partial}{\partial x_{2 j-1}}\right]
$$

гамильтоново векторное поле, и пусть $Y$ - произвольное вещественноаналитическое векторное поле, касаюшееся гиперповерхности $V=f^{-1}(0)$, c $h:=(d f / f)(Y)$. Для семейства векторньх полей $X_{t}:=H+t Y(t \in \mathbb{R}$, $|t|<\varepsilon,|x|<\delta)$ имеем

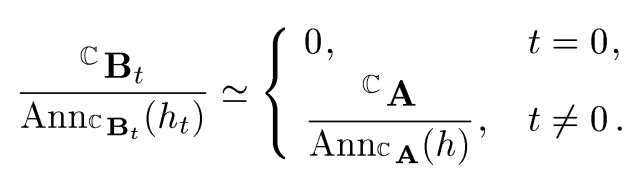


Для $t \neq 0$ билинейнье формы, определеннье относительным якобиевьм определителем $J_{f} X_{t}$ на $\left[{ }^{\mathbb{C}} \mathbf{B}_{t} / \operatorname{Ann}_{\mathbf{B}_{t}}\left(h_{t}\right)\right]_{\mathbb{R}}^{+}$, соответствуют билинейньлм формам, определенньм относительным гессианом $t \operatorname{Hess}_{\mathrm{rel}}(h) \in \mathbf{A} / \operatorname{Ann}_{\mathbf{A}}(h)$.

ДокАЗАТЕльство. В дополнение к последовательности (4.1), полученной умножением на $h_{t}=t h$, рассмотрим последовательность, полученную умножением на $h$,

$$
0 \rightarrow\left\{\frac{\operatorname{Ann}_{\mathbf{B}_{t}}(h)}{(f)}\right\} \rightarrow\left\{\frac{{ }^{\mathbb{C}} \mathbf{B}_{t}}{(f)}\right\} \stackrel{h}{\rightarrow}\left\{\operatorname{Ann}_{\mathbb{C} \mathbf{B}_{t}}(f)\right\} \rightarrow\left\{\frac{\operatorname{Ann}_{\mathbf{B}_{t}}(f)}{(h)}\right\} \rightarrow 0 .
$$

Ядра и образы в последовательности (4.4) образуют векторные подрасслоения:

а) для $t \neq 0$ в соответствии с (3.3) последовательности (4.4) и (4.1) имеют совпадающие ядра и коядра комплексной размерности $\operatorname{dim}_{\mathbb{R}}\left(\operatorname{Ann}_{\mathbf{A}}(h) /(f)\right)$;

b) для $t=0$ имеем $\mathbf{B}_{0}={ }^{\mathbb{C}} \mathbf{A}$, и поэтому ядра и коядра в $(4.4)-$ это $\operatorname{Ann}_{\mathbb{C}_{\mathbf{A}}}(h) /(f)$ и $\operatorname{Ann}_{\mathbb{C}_{\mathbf{A}}}(f) /(h)$ соответственно.

Таким образом, семейство алгебр ${ }^{\mathbb{C}} \mathbf{B}_{t} / \operatorname{Ann}_{\mathbb{C}_{t}}(h)$, полученное в середине последовательности (4.4), образует комплексное векторное расслоение, а множество $\left[{ }^{\mathbb{C}} \mathbf{B}_{t} / \operatorname{Ann}_{\mathbf{B}_{t}}(h)\right]_{\mathbb{R}}^{+}(t \in \mathbb{R})$ неподвижных точек комплексного сопряжения в ${ }^{\mathbb{C}} \mathbf{B}_{t} / \operatorname{Ann}_{\mathbb{C}_{t}}(h)(t \in \mathbb{R})$ образует вещественное векторное расслоение. В последнем векторном расслоении можно рассмотреть невырожденную двойственность, полученную, как в (2), выбором $J X_{t} / h$ в качестве образующей его одномерного цоколя. В частности, все эти билинейные формы имеют одну и ту же сигнатуру. Мы вычисляем эту обшую сигнатуру в точке $t=0$, где $\mathbf{B}_{0}=\mathbf{A}$ и $J X_{0}=\operatorname{Hess}(f)$. Отсюда вытекает, что сигнатура алгебры $\mathbf{A} / \operatorname{Ann}_{\mathbf{A}}(h)$, индуцированная относительным гессианом $\operatorname{Hess}(f) / h$, совпадает с сигнатурой формы на алгебре $\left[{ }^{\mathbb{C}} \mathbf{B}_{t} / \operatorname{Ann}_{\mathbb{C}} \mathbf{B}_{t}(h)\right]_{\mathbb{R}}^{+}$, индуцированной относительным якобианом $J X_{t} /(t h)$ для $t>0$. Это доказывает (4.3) для $t>0$. Для $t<0$ происходит изменение знака и эта сигнатура равна взятой со знаком минус сигнатуре алгебры $\mathbf{A} / \operatorname{Ann}_{\mathbf{A}}(h)$, индуцированной относительным гессианом $\operatorname{Hess}_{\mathbf{A}}(-h)$.

ЛЕмма 4.2. Для вещественно-аналитического векторного поля $X_{0}$ на $B \subset \mathbb{R}^{n+1}$, касающегося $V=f^{-1}(0)$ и имеющего алгебраически изолированную особенность в начале координат 0 , существует сколь угодно близкое $к$ $X_{0}$ вещественно-аналитическое векторное поле $X_{1}$ на $B$, касающееся $V u$ такое, что векторные поля $H+t\left(X_{1}-H\right)(t \in[0,1])$ имеют алгебраически изолированнье особенности в начале координат, где $H$ - гамильтоново векторное поле Функиии $f$.

ДокАЗАтЕЛьство. Можно найти голоморфные векторные поля $W_{1}, \ldots$, $W_{m-2}$ на $B$, касающиеся $V$ и такие, что $\delta_{f}\left(W_{j}\right)=0$, а векторы $W_{1}(p), \ldots$, $W_{m-2}(p)$ порождают касательное пространство $T_{p} B$ для любой точки $p \in$ $B \backslash V$ и пространство $T_{p} V$ для любой точки $p \in V \backslash\{0\}$. Это следует из теоремы А Картана [9] и из того факта, что пучок векторных полей с $\delta=0$ является когерентным. Предположим также, что по крайней мере одно из векторных полей $W_{j}$ имеет особую точку $p \in B \backslash V$ индекса 1 , а другое векторное поле $W_{k}$ имеет особую точку $q \in V \backslash\{0\}$ индекса 1 . Добавим к этому семейству $W_{m-1}=H$ и $W_{m}=X_{0}$. Векторное пространство $V$ голоморфных 
векторных полей, порожденное полями $W_{1}, \ldots, W_{m}$, параметризует линейное семейство векторных полей. Рассуждая теперь, как в лемме 7.іi из [7], получаем, что имеется аналитическое подпространство $\mathrm{Land}^{2} \subset W$ коразмерности, большей, чем 2 , которого следует избегать. Первое условие, определяющее Land $^{2}$, состоит в том, что «внешний нуль попадает в 0». Второе условие состоит в том, что «этот внешний нуль, попадающий в 0 , имеет кратность как минимум 2 ». Заметим, что $\delta_{f}$, ограниченное на $V$, вследствие его линейности принимает значения в $\mathbb{R} \delta_{f}\left(X_{0}\right)$, так как $\delta_{f}$ обращается в нуль на образующих $W_{j}, j \neq n$. Следовательно, $\delta_{f}^{-1} \delta_{f}\left(X_{0}\right) \subset V$ имеет коразмерность 1 в $V$. Отсюда вытекает, что проекция множества $\mathrm{Land}^{2} \subset V$ из центра $H$ на экран $\delta_{f}^{-1}\left(\delta_{f}\left(X_{0}\right)\right)$ не может заполнять окрестность векторного поля $X_{0}$ на экране проекции (так как это проекция из точки множества, вещественная коразмерность которого не меньше, чем 2). Точка $X_{1}$ на экране, не принадлежащая проекции множества Land ${ }^{2}$, и является требуемой.

ДокАЗАтЕЛЬСтво теоремы 1. Пусть $X \in \mathfrak{X}(V)$ - векторное поле, описанное в теореме. Для векторных полей $Y \in \mathfrak{X}(V)$, близких к $X$, имеется целозначная функция $k_{X}(Y)$, определенная формулой

$$
\operatorname{Sgn}_{V, 0}(X)=\operatorname{Sgn}_{V, 0}(Y)+\sum_{\substack{Y_{t}(p)=0, f(p)=0, p \in \mathbb{R}^{n} \backslash\{0\}}} \operatorname{Ind}_{V, p}\left(\left.Y\right|_{V}\right)+k_{X}(Y) .
$$

В соответствии с предложением 3.1 значение функции $k_{X}(Y)$ зависит только от связного страта $\delta_{f}^{-1}\left(V_{j}\right)$, которому принадлежит $Y$. Если мы изменяем $X$ в пределах его страта, функция $k_{X}(Y)$ остается той же. Следовательно, по лемме 4.2 для того, чтобы вычислить $k_{X}(Y)$, можно предположить, что $X$ сколь угодно близко к гамильтонову векторному полю.

В предложении 4.1 мы уже вычислили описанную выше функцию $k_{H}(Y)=$ $\operatorname{Sgn}_{\mathbf{A}}(\delta(Y))$ для гамильтонова векторного поля. Из этой формулы и предложения 3.1 формула (5) вытекает немедленно.

Tак как функция индекса $\operatorname{Ind}_{V, \pm, 0}$ удовлетворяет закону сохранения, аналогичному формуле (5) (см. [6]), то для некоторой константы $K$ выполняется равенство

$$
\operatorname{Ind}_{V, \pm, 0}(X)=\operatorname{Sgn}_{V, 0}(X)-\operatorname{Sgn}_{\mathbf{A}}\left(\frac{d f}{f}(X)\right)+K \quad \forall X \in \mathfrak{X}(V) .
$$

Для того чтобы закончить доказательство теоремы 1, остается показать, что константа $K$ равна нулю. Чтобы сделать это, вычислим (4.6) на гамильтоновом векторном поле, для которого мы знаем, что $\operatorname{Ind}_{V, \pm, 0}(H)=0$, поскольку $\left.H\right|_{V_{ \pm \varepsilon}}$ касается гиперповерхности $V_{ \pm \varepsilon}$ и не обрашается в нуль на $V_{ \pm \varepsilon}$. Поскольку $(d f / f)(H)=0$, из (4.6) мы получаем, что

$$
0=\operatorname{Sgn}_{V, 0}(H)-\operatorname{Sgn}_{\mathbf{A}}(H)+K=0-0+K .
$$

Следовательно, $K=0$.

ПримеР 4.3. Рассмотрим вещественную алгебраическую кривую $V \subset \mathbb{R}^{2}$, определенную, как в примере 1.3 , ростком $f=y^{3}-x^{2}$, и векторные поля

$$
L=3 x \frac{\partial}{\partial x}+2 y \frac{\partial}{\partial y}, \quad H=3 y^{2} \frac{\partial}{\partial x}+2 x \frac{\partial}{\partial y}, \quad W=y L+f \frac{\partial}{\partial x}
$$


на плоскости $\mathbb{R}^{2}$. Поле $L$ является линейным векторным полем, а $H$ - гамильтоновым. Рассмотрим семейство векторных полей

$$
\begin{aligned}
X_{t} & :=t_{1} L+t_{2} H+t_{3} W \\
& =\left(3 x t_{1}+3 y^{2} t_{2}+\left(3 x y+y^{3}-x^{2}\right) t_{3}\right) \frac{\partial}{\partial x}+\left(2 y t_{1}+2 x t_{2}+2 y^{2} t_{3}\right) \frac{\partial}{\partial y}, \\
h_{t} & :=\frac{d f}{f}\left(X_{t}\right)=6 t_{1}+(6 y-2 x) t_{3}, \quad t=\left(t_{1}, t_{2}, t_{3}\right) \in \mathbb{R}^{3} \backslash\{0\} .
\end{aligned}
$$

Все эти поля имеют изолированные особенности и касаются $V$. Мы хотим показать, что

$$
\begin{aligned}
\operatorname{Ind}_{V, 0}\left(X_{t}\right)=\left\{\begin{aligned}
+1, & t_{1}>0, \\
-1, & t_{1}<0, \\
0, & t_{1}=0, t_{2} \neq 0, \\
+1, & t_{1}=t_{2}=0, t_{3}>0, \\
-1, & t_{1}=t_{2}=0, t_{3}<0,
\end{aligned}\right. \\
\operatorname{Sgn}_{V, 0}\left(X_{t}\right)=\left\{\begin{array}{cl}
+1, & t_{1}>0, \\
-1, & t_{1}<0, \\
-1, & t_{1}=0, t_{2} \neq 0, t_{3}>0, \\
0, & t_{1}=0, t_{2} \neq 0, t_{3}=0, \\
+1, & t_{1}=0, t_{2} \neq 0, t_{3}<0, \\
0, & t_{1}=t_{2}=0, t_{3} \neq 0, \\
-1, & t_{1}=0, t_{3}>0, \\
+1, & t_{1}=0, t_{3}<0, \\
0 & \text { в противном случае, }
\end{array}\right.
\end{aligned}
$$

и тем самым получить явное доказательство формулы (4) для этого семейства; кроме того, рассмотрев семейство $H+t_{3} W$, мы можем убедиться в необходимости обоих членов в (4).

Простейший способ проверить первую формулу в (4.7) — это использовать параметризацию $s \rightarrow\left(s^{3}, s^{2}\right)=(x, y)$ кривой $V$, задаваемую нормализацией, для которой непосредственно проверяется, что векторное поле $t_{1} L+t_{2} H+t_{3} W$ переходит в $Y_{t}=\left(t_{1} s+t_{2} s^{2}+t_{3} s^{3}\right) \partial / \partial s$. Вычисление индекса векторного поля $Y_{t}$ в начале координат 0 элементарно (надо использовать [5] или [10]) и дает первую формулу в (4.7). Индексы, полученные с использованием сглаживания слоя Милнора и с использованием нормализации, совпадают, поскольку обе модели представляют собой интервал (см. [3]).

Опишем поведение относительной сигнатуры для $X_{t}$. Семейство алгебр, соответствуюших особой точке 0 полей $X_{t}$ на плоскости $\mathbb{C}^{2}$, - это

$$
\mathbf{B}_{t, 0}=\frac{\mathscr{O}_{\mathbb{C}^{2}, 0}}{\left(3 x t_{1}+3 y^{2} t_{2}+\left(3 x y+y^{3}-x^{2}\right) t_{3}, 2 y t_{1}+2 x t_{2}+2 y^{2} t_{3}\right)},
$$

а якобиев определитель равен

$$
J X_{t}=6 t_{1}^{2}+\left(-4 t_{1} t_{3}-6 t_{2} t_{3}\right) x+\left(-12 t_{2}^{2}+18 t_{1} t_{3}\right) y+\left(12 t_{3}^{2}-6 t_{2} t_{3}\right) y^{2}-8 t_{3}^{2} x y .
$$

Рассмотрим различные случаи:

1) Если $t_{1} \neq 0$, то

$$
\mathbf{B}_{t, 0} \simeq \mathbb{R}, \quad h_{t}=6 t_{1}, \quad \frac{\mathbf{B}_{t, 0}}{\operatorname{Ann}_{\mathbf{B}_{t, 0}}\left(h_{t}\right)} \simeq \mathbb{R}, \quad J_{f} X_{t}=\frac{6 t_{1}^{2}}{6 t_{1}}=t_{1},
$$


откуда следует, что билинейные формы, определенные, как в (2), относительным якобианом, имеют сигнатуру, равную 1 при $t_{1}>0$ и -1 при $t_{1}<0$.

2) Если $t_{1}=0, t_{2} \neq 0$, то $\mathbf{B}_{t, 0} \simeq \mathbb{R} \oplus \mathbb{R} y, h_{t}=6 t_{3} y$. Здесь имеются два подслучая:

a) если $t_{3} \neq 0$, то $\mathbf{B}_{t, 0} / \operatorname{Ann}_{\mathbf{B}_{t, 0}}\left(h_{t}\right) \simeq \mathbb{R}, J_{f} X_{t}=-2 t_{2}^{2} / t_{3}$, откуда следует, что билинейные формы, определенные, как в (2), относительным якобианом, имеют сигнатуру, равную -1 при $t_{3}>0$ и +1 при $t_{3}<0$;

b) если $t_{3}=0$, то $\mathbf{B}_{t, 0} / \operatorname{Ann}_{\mathbf{B}_{t, 0}}\left(h_{t}\right) \simeq 0$, откуда следует, что в этом случае сигнатура равна 0 .

3) Если $t_{1}=t_{2}=0$, но $t_{3} \neq 0$, то

$$
\begin{gathered}
\mathbf{B}_{t, 0} \simeq \mathbb{R} \oplus \mathbb{R} x \oplus \mathbb{R} y \oplus \mathbb{R} x^{2}, \quad y^{2}=0, \quad 3 x y=x^{2}, \\
h_{t}=(6 y-2 x) t_{3}, \quad \frac{\mathbf{B}_{t, 0}}{\operatorname{Ann}_{\mathbf{B}_{t, 0}}\left(h_{t}\right)} \simeq \mathbb{R} \oplus \mathbb{R} y, \quad J_{f} X_{t}=4 t_{3} y,
\end{gathered}
$$

откуда следует, что билинейные формы, определенные, как в (2), относительным якобианом, имеют в этом случае сигнатуру, равную 0 .

Мы уже вычислили (4.5) в (1.3), где $\delta_{f}\left(X_{t}\right)=6 t_{1}+6 t_{3} y \in \mathbf{A}=\mathbb{R} \oplus \mathbb{R} y$.

\section{ЛИТЕРАТУРА}

1. Арнольд В. И. Индекс особой точки векторного поля, неравенства Петровского-Олейник и смешанные структуры Ходжа. Функц. анализ и его прил., 12, No. 1, 1-14 (1978).

2. Арнольд В. И., Варченко А. Н., Гусейн-Заде С. М. Особенности дифференцируемых отображений, т. I. Наука, М., 1982.

3. Bonatti Ch., Gómez-Mont X. The index of a holomorphic vector field on a singular variety I. Asterisque, 222, 9-35 (1994).

4. Ebeling W., Gusein-Zade S. M. On the index of a vector field at an isolated singularity. Preprint.

5. Eisenbud D., Levine $H$. An algebraic formula for the degree of a $C^{\infty}$ map germ. Ann. of Math., 106, 19-38 (1977).

6. Gómez-Mont X. An algebraic formula for the index of a vector field on a hypersurface with an isolated singularity. Preprint Comunicaciones del CIMAT I-96-27, 1996, pp. 1-23.

7. Gómez-Mont X., Mardešić P. The index of a vector field tangent to a hypersurface and the signature of the relative Jacobian determinant. Ann. Inst. Fourier (Grenoble), 47, No. 5 (1997).

8. Gómez-Mont X., Seade J., Verjovsky A. The index of a holomorphic flow with an isolated singularity. Math. Ann., 291, 737-751 (1991).

9. Grauert H., Remmert H. Coherent analytic sheaves. Grundlehren 265, SpringerVerlag, 1984.

10. Химшиашвили Г. Н. О локальной степени гладкого отображения. Сообщ. АН ГрузССР, 85, № 2, 309-311 (1977).

CIMAT, A. P. 402, Guanajuato, 36000, México e-mail: gmont@fractal.cimat.mx

Поступило в редакцию 30 ноября 1997 г.

Laboratoire de Topologie UMR $5584 \mathrm{du}$ CNRS,

U. Bourgogne, BP 400, 21011-Dijon, France

e-mail: mardesic@u-bourgogne.fr 